\title{
PARAMETERS SET EVALUATION OF WIENER MODELS FROM DATA WITH BOUNDED OUTPUT ERRORS
}

\author{
V. Cerone M. Milanese D. Regruto
}

Dipartimento di Automatica e Informatica, Politecnico di Torino, corso Duca degli Abruzzi 24, 10129 Torino, Italy - E-mail: vito.cerone@polito.it, mario.milanese@polito.it, diego.regruto@polito.it, Phone: + 39115647064 , Fax: + 39115647099

\begin{abstract}
In this paper a procedure is presented for deriving parameters bounds in SISO Wiener models when the nonlinear block can be modeled by a polynomial and the output measurement errors are bounded. First, using steady-state input-output data, parameters of the nonlinear block are tightly bounded. Next in order to estimate the parameters of the linear block, the evaluation of the inner unmeasurable signal is considered. No invertibility assumption of the nonlinearity is required. Then, through a suitable design of the identification experiment, bounds on the unmeasurable inner signal are evaluated. Finally, such bounds together with the input sequence are used for bounding the parameters of the linear model.
\end{abstract}

Keywords: Wiener model, bounded uncertainty, output errors, parameter bounding, linear programming.

\section{INTRODUCTION}

A wide class of simple nonlinear systems, also called block-oriented systems, can be modeled by interconnected memoryless nonlinear gains and linear subsystems. The configuration considered in this paper, commonly referred to as Wiener model, is shown in Figure 1; it consists of a linear dynamic system followed by static nonlinear block $\mathcal{N}$. The identification of such a model is carried out on the basis of the sequences $u_{t}$ and $y_{t}$, while the inner signal $x_{t}$, i.e. the output of the linear block, is not assumed to be available. The Wiener model has been successfully used in a large variety of fields. The identification of Wiener structure has attracted the attention of many authors, as can be seen in Billings (1980), Billings and Fakhouri (1977), Haber and Unbehauen (1990), Bai (2002), Wigren (1998). The main difficulty in the identification of nonlinear block-oriented system is that the internal signals are not available for measurement. As far as Wiener systems are concerned most of contributions assume invertibility of the nonlinearity. As a matter of fact under such an assumption the inner signals can be recovered from the output measurements through inversion of the previously estimated nonlinearity.
However, many output nonlinearity encountered in real world problem are non-invertible (see, e.g., Wigren, 1998), thus the invertibility assumption appear to be quite restrictive. Removal of such an hypothesis made the consistent evaluation of the inner signal sequence a difficult task even in the case of exactly known nonlinearity. In all the papers mentioned above, the authors assume that the measurement error $\eta_{t}$ is statistically described. A worthwhile alternative to the stochastic description of measurement errors is the bounded-errors characterization, where uncertainties are assumed to belong to a given set. The interested reader can find further details on this approach in a number of survey papers (see, e.g., (Milanese and Vicino, 1991; Walter and Piet-Lahanier, 1990)), in the book edited by Milanese et al. (Milanese et al., 1996) and the special issues edited by Norton (Norton(Ed.), 1994; Norton(Ed.), 1995). To the author's best knowledge, no contribution can be found which address the identification of Wiener models when the measurement error $\eta_{t}$ is supposed to be bounded. In this paper the identification of single-input single-output (SISO) Wiener models is considered, when the nonlinear block can be modeled by a polynomial, with finite and known order, and when the output mea- 
surement errors are bounded. First, using steadystate input-output data, parameters of the nonlinear block are tightly bounded. Next in order to estimate the parameters of the linear block, the evaluation of the inner unmeasurable signal $x_{t}$ is considered. No invertibility assumption of the nonlinearity is required, thus the inner signal $x_{t}$ providing a given measured output $y_{t}$ is not unique even in the uncertainty free case, i.e., the estimated inner sequence $\left\{x_{t}\right\}$ to be used in the evaluation of the feasible parameter set of the linear block, might not be the output of a unique linear systems. In the paper it is shown how such a fundamental problem can be addressed through a suitable design of the identification experiment. Finally, through the designed dynamic experiment, for all $y_{t}$ belonging to a given output transient sequence $\left\{y_{t}\right\}$, upper and lower bounds on the inner signal $x_{t}$ are computed. Such bounds, together with the input sequence $\left\{u_{t}\right\}$ are used for bounding the parameters of the linear block.

\section{PROBLEM FORMULATION}

Consider the SISO discrete-time Wiener model shown in Figure 1, where the linear dynamic block, which is modeled by a discrete-time system, maps the input signal $u_{t}$ into the unmeasurable inner variable $x_{t}$ according to

$$
x_{t}=\frac{B\left(q^{-1}\right)}{A\left(q^{-1}\right)} u_{t}=G\left(q^{-1}\right) u_{t},
$$

or, equivalently, in terms of a linear difference equation

$$
A\left(q^{-1}\right) x_{t}=B\left(q^{-1}\right) u_{t},
$$

where $A(\cdot)$ and $B(\cdot)$ are polynomials in the backward shift operator $q^{-1},\left(q^{-1} w_{t}=w_{t-1}\right)$,

$$
\begin{aligned}
& A\left(q^{-1}\right)=1+a_{1} q^{-1}+\ldots+a_{n a} q^{-n a}, \\
& B\left(q^{-1}\right)=b_{0}+b_{1} q^{-1}+\ldots+b_{n b} q^{-n b} .
\end{aligned}
$$

The nonlinear block transforms $x_{t}$ into the noisefree output $w_{t}$ through the following polynomial function

$$
w_{t}=\sum_{k=1}^{n} \gamma_{k} x_{t}^{k}, \quad t=1, \ldots, N ;
$$

whose order $n$ is taken to be finite and a-priori known; $N$ is the length of the input sequence. In line with the work done by a number of authors, it is assumed that (i) the linear system is asymptotically stable (see, e.g. Stoica and Söderström, 1982; Krzyżak, 1993; Lang, 1993; Sun et al., 1999); (ii) $\sum_{j=0}^{n b} b_{j} \neq 0$, that is, the steadystate gain is not zero (see, e.g. Lang, 1993; Sun et al., 1999); (iii) the only a priori information needed is an estimate of the process settling-time (see, e.g. Kalafatis et al., 1997). Let $y_{t}$ be the noise-corrupted measurements of $w_{t}$

$$
y_{t}=w_{t}+\eta_{t} .
$$

Measurements uncertainty is known to range within given bounds $\Delta \eta_{t}$, i.e.,

$$
\left|\eta_{t}\right| \leq \Delta \eta_{t}
$$

Unknown parameter vectors $\gamma \in R^{n}$ and $\theta \in R^{p}$ are defined, respectively, as

$$
\begin{gathered}
\gamma^{T}=\left[\begin{array}{lllll}
\gamma_{1} & \gamma_{2} & \ldots & \gamma_{n}
\end{array}\right], \\
\theta^{T}=\left[\begin{array}{lllllll}
a_{1} & \ldots & a_{n a} & b_{0} & b_{1} & \ldots & b_{n b}
\end{array}\right],
\end{gathered}
$$

where $n_{a}+n_{b}+1=p$. It is easy to show that the parameterization of the structure of Figure 1 is not unique. As a matter of fact, any parameters set $\tilde{b}_{j}=\alpha^{-1} b_{j}, j=1,2, \ldots, n b$, and $\tilde{\gamma}_{k}=\alpha \gamma_{k}, k=1,2, \ldots, n$, for some nonzero and finite constant $\alpha$, provides the same inputoutput behaviour. Thus, any identification procedure cannot perceive the difference between parameters $\left\{b_{j}, \gamma_{k}\right\}$ and $\left\{\alpha^{-1} b_{j}, \alpha \gamma_{k}\right\}$. In this work, it is assumed, without loss of generality, that the steady-state gain of the linear part be one, that is

$$
g=\frac{\sum_{j=0}^{n b} b_{j}}{1+\sum_{i=1}^{n a} a_{i}}=1
$$

In this paper the problem of deriving bounds on parameters $\gamma$ and $\theta$ consistently with given measurements, error bounds and the assumed model structure is addressed.

\section{ASSESSMENT OF TIGHT BOUNDS ON THE NONLINEAR STATIC BLOCK PARAMETERS}

In this work we exploit steady-state operating conditions to bound the parameters of the nonlinear static block. The known input and noise corrupted output sequences are collected from the steady-state response of the system to a set of step inputs with different amplitude. It is only assumed that a rough information on the settling time of the system under consideration is available, in order to know when steady-state conditions are reached, so that steady-state data can be collected. Indeed, combining equations (5), (2), (6) and (10) in steady-state operating conditions the following input-output description is obtained.

$$
\bar{y}_{s}=\sum_{k=1}^{n} \gamma_{k} \bar{u}_{s}^{k}+\eta_{s}, \quad s=1, \ldots, M
$$

where $\bar{u}_{s}$ and $\bar{y}_{s}$ are steady-state values of the known input signal and output observation respectively, while $\eta_{s}$ is the measurement error; $M \geq n$ is the length of the steady-state sequences. A block diagram description of equation (11) is depicted in Figure 2. Thus, the feasible parameter region of the static nonlinear block is defined as

$$
\begin{gathered}
\mathcal{D}_{\gamma}=\left\{\gamma \in R^{n}: \bar{y}_{s}=\sum_{k=1}^{n} \gamma_{k} \bar{u}_{s}^{k}+\eta_{s},\right. \\
\left.\left|\eta_{s}\right| \leq \Delta \eta_{s} ; \quad s=1, \ldots, M\right\} .
\end{gathered}
$$

where $\left\{\Delta \eta_{s}\right\}$ is the sequence of bounds on measurements uncertainty. From the definition of $\mathcal{D}_{\gamma}$ one gets

$$
\begin{aligned}
& \bar{\varphi}_{s}^{\mathrm{T}} \gamma \leq \bar{y}_{s}+\Delta \eta_{s} \\
& \bar{\varphi}_{s}^{\mathrm{T}} \gamma \geq \bar{y}_{s}-\Delta \eta_{s}
\end{aligned}
$$

where 


$$
\bar{\varphi}_{s}=\left[\begin{array}{lll}
\bar{u}_{s} & \bar{u}_{s}^{2} & \bar{u}_{s}^{3} \ldots \bar{u}_{s}^{n}
\end{array}\right]^{\mathrm{T}}
$$

for $s=1,2, \ldots, M$. The above exact description of $\mathcal{D}_{\gamma}$ will be used in the next section when deriving tight bounds on the unmeasurable inner signal $x_{t}$. Since $\mathcal{D}_{\gamma}$ is a convex polytope, whose shape may result quite complex for increasing $n$ and $M$, an outer bound to it such as an ellipsoid or a box is often computed. In this paper we consider an orthotope-outer bounding set $\mathcal{B}_{\gamma}$ containing $\mathcal{D}_{\gamma}$

$$
\begin{gathered}
\mathcal{B}_{\gamma}=\left\{\gamma \in R^{n}: \gamma_{j}=\gamma_{j}^{c}+\delta \gamma_{j},\right. \\
\left.\left|\delta \gamma_{j}\right| \leq \Delta \gamma_{j} / 2, j=1, \ldots, n\right\},
\end{gathered}
$$

where

$$
\begin{gathered}
\gamma_{j}^{c}=\frac{\gamma_{j}^{\text {min }}+\gamma_{j}^{\max }}{2}, \\
\Delta \gamma_{j}=\left|\gamma_{j}^{\max }-\gamma_{j}^{\text {min }}\right|,
\end{gathered}
$$

and

$$
\gamma_{j}^{\min }=\min _{\gamma \in \mathcal{D}_{\gamma}} \gamma_{j}, \quad \gamma_{j}^{\max }=\max _{\gamma \in \mathcal{D}_{\gamma}} \gamma_{j} .
$$

The evaluation of $\mathcal{B}_{\gamma}$ requires the solution of $2 n$ linear programming problems with $n$ variables and $2 M$ constraints.

\section{EVALUATION OF FEASIBLE INTERVALS FOR OUTPUT MEASUREMENTS AND INNER SIGNALS}

In the previous section it has been shown how the nonlinear block can be characterized using steadystate data. In order to estimate the parameters of the linear model, one should first evaluate the inner signal $x_{t}$ from the output records $y_{t}$ via the polynomial nonlinearity. Unfortunately, one must consider the fact that nonlinearity (5) is, in general, noninvertible, which means that, given the measured output $y_{t}$, the inner signal $x_{t}$ cannot be evaluated uniquely even in the case of exactly known polynomial and noise free measurements. As a matter of fact, considering (5) at a single time $t$ one gets

$$
p_{t}\left(x_{t}, w_{t}\right)=0
$$

where

$$
p_{t}\left(x_{t}, w_{t}\right)=w_{t}-\sum_{k=1}^{n} \gamma_{k} x_{t}^{k} .
$$

belongs to the following family of polynomials

$$
\mathcal{P}_{t}=\left\{p_{t}\left(x_{t}, w_{t}\right): w_{t} \in R\right\}
$$

For given $w_{t}$ and exactly known $\gamma_{k}, k=$ $1,2, \ldots, n$, equation (20) shows, in general, $n$ different solutions in the unknown $x_{t}$. Nonuniqueness, unfortunately, is responsible of nonconsistent inner signal estimates, i.e., estimated $\left\{x_{t}\right\}$ might not be the output of a unique linear system. The main idea for overcoming this problem is to design the input sequence $\left\{u_{t}\right\}$ which will force an output sequence $\left\{w_{t}\right\}$ so that polynomial (21) will show either only one real root when $n$ is odd or two real roots when $n$ is even in the case of exactly known parameters of the nonlinear block and noiseless measurements. In the case of uncertain polynomial parameters the following family of polynomials can be defined

$$
\Pi_{t}=\left\{p_{t}\left(x_{t}, w_{t}, \gamma\right): w_{t} \in R, \gamma \in \mathcal{D}_{\gamma}\right\}
$$

where

$$
p_{t}\left(x_{t}, w_{t}, \gamma\right)=w_{t}-\sum_{k=1}^{n} \gamma_{k} x_{t}^{k}
$$

It is assumed that all polynomials in $\Pi_{t}$ have degree equal to $n$. In this case, in order to evaluate the inner signal $x_{t}$ one has to find the real roots of the uncertain polynomial (24). The real spectral set of the family of polynomial $\Pi_{t}$, is defined as

$$
\begin{gathered}
\mathcal{S}_{\mathrm{R}}\left(\Pi_{t}\right)=\left\{x_{t} \in R: p_{t}\left(x_{t}, w_{t}, \gamma\right)=0\right. \\
\text { for some } \left.\gamma \in \mathcal{D}_{\gamma}, w_{t} \in R\right\}
\end{gathered}
$$

4.1 Uncertain polynomial nonlinearity - Here it is assumed that $\gamma \in \mathcal{D}_{\gamma}$ and the problem of evaluating the inner signal $x_{t}$ in terms of roots of the uncertain polynomial (24) is addressed. One shall look for conditions under which each polynomial $p_{t}\left(x_{t}, w_{t}, \gamma\right) \in \Pi_{t}$ shows either only one real root when $n$ is odd or two real roots when $n$ is even.

Proposition 1. Each polynomial $p_{t}\left(x_{t}, w_{t}, \gamma\right) \in$ $\Pi_{t}$, shows either only one real root when $n$ is odd or two real roots when $n$ is even if and only if

$$
w_{t}>\bar{w} \text { or } w_{t}<\underline{w} \text { when } n \text { is odd }
$$

or

$$
\operatorname{sign}\left(\gamma_{n}\right) w_{t}>\frac{1+\operatorname{sign}\left(\gamma_{n}\right)}{2} \bar{w}-\frac{1-\operatorname{sign}\left(\gamma_{n}\right)}{2} \underline{w}
$$

when $n$ is even

where

$$
\begin{aligned}
& \bar{w}=\max _{x_{t} \in \Upsilon_{t}} \max _{\gamma \in \mathcal{D}_{\gamma}} \sum_{k=1}^{n} \gamma_{k} x_{t}^{k} \\
& \underline{w}=\min _{x_{t} \in \Upsilon_{t}} \min _{\gamma \in \mathcal{D}_{\gamma}} \sum_{k=1}^{n} \gamma_{k} x_{t}^{k}
\end{aligned}
$$

$\Upsilon_{t}=\left\{x_{t} \in R: \frac{d}{d x_{t}} \sum_{k=1}^{n} \gamma_{k} x_{t}^{k}=0\right.$, for some $\left.\gamma \in \mathcal{D}_{\gamma}\right\}$

Moreover, under conditions (26) and (27) the real roots of polynomial (24) satisfy

$$
\begin{gathered}
\left.\left.x_{t} \in\right] \bar{x},+\infty\right] \\
x_{t} \in[-\infty, \underline{x}[ \\
\bar{x}=\max \left\{x_{t} \in R: \frac{1+\operatorname{sign}\left(\gamma_{n}\right)}{2} \bar{w}+\right. \\
\frac{1-\operatorname{sign}\left(\gamma_{n}\right)}{2} \underline{w}-\sum_{k=1}^{n} \gamma_{k} x_{t}^{k}=0, \\
\text { for some } \left.\gamma \in \mathcal{D}_{\gamma}\right\} \\
\underline{x}=\min \left\{x_{t} \in R: \frac{1+(-1)^{n} \operatorname{sign}\left(\gamma_{n}\right)}{2} \bar{w}+\right. \\
\frac{1-(-1)^{n} \operatorname{sign}\left(\gamma_{n}\right)}{2} \underline{w}-\sum_{k=1}^{n} \gamma_{k} x_{t}^{k}=0,
\end{gathered}
$$

for some $\left.\gamma \in \mathcal{D}_{\gamma}\right\}$.

The proof of Proposition 1 can be found in (Cerone et al., 2002). 
The computational aspects of quantities and sets involved in Proposition 1 are now discussed.

Computation of $\Upsilon_{t}$ - First consider the set defined by equation (30), i.e. the set of real valued $x_{t}$ for which the uncertain polynomial shows stationary points (relative maxima, relative minima or points of inflexion). The first derivative of the uncertain polynomial is still an uncertain polynomial, namely

$$
p_{t}{ }^{\prime}\left(x_{t}, \gamma\right)=-\frac{d}{d x_{t}} \sum_{k=1}^{n} \gamma_{k} x_{t}^{k}=-\sum_{k=1}^{n} k \gamma_{k} x_{t}^{k-1}
$$

which, clearly, shows nonlinear relations in the unknown $x_{t}$ and the uncertain $\gamma$. A one-dimensional gridding procedure for finding the roots of $(35)$ is proposed. It is noticed that for a given $x_{t} \in R$, in order to find the real spectral set of polynomial (35) one must solve a set of $2 M$ linear inequalities (i.e., $\gamma \in \mathcal{D}_{\gamma}$ ) and one linear equality (i.e. $\left.\sum_{k=1}^{n} k \gamma_{k} x_{t}^{k-1}=0\right)$ in the unknown $\gamma \in R^{n}$.

Computation of $\bar{w}$ and $\underline{w}$ - Next equations (28) and (29) which define two nonlinear programming problems are considered. However, when $x_{t}$ is given, they simplify to linear programs. Thus, to compute $\bar{w}$ and $\underline{w}$, for each value of $x_{t} \in \Upsilon_{t}$ the solution of two linear programming problems with $n$ variables and $2 M$ constraints is required. A onedimensional gridding procedure is used in order to carry out the optimization over a finite number of $x_{t} \in \Upsilon_{t}$.

Computation of $\bar{x}$ and $\underline{x}$ - Finally, equation (33) and equation (34) are considered. In order to simplify the discussion, odd order polynomial with $\gamma_{n}>0$ are first considered. In this case one gets

$$
\begin{aligned}
& \bar{x}=\max \left\{x_{t} \in R: \bar{w}-\sum_{k=1}^{n} \gamma_{k} x_{t}^{k}=0,\right. \\
& \text { for some } \left.\gamma \in \mathcal{D}_{\gamma}\right\} \\
& \underline{x}=\min \left\{x_{t} \in R: \underline{w}-\sum_{k=1}^{n} \gamma_{k} x_{t}^{k}=0,\right. \\
& \text { for some } \left.\gamma \in \mathcal{D}_{\gamma}\right\}
\end{aligned}
$$

Equations (36) and (37) show nonlinear relations in the unknown $x_{t}$ and the uncertain $\gamma$. In this case a search procedure is proposed for finding the roots of uncertain polynomials involved in the above equations. It is noticed that for a given $x_{t} \in R$, in order to solve each one of the above equations one must solve a set of $2 M$ linear inequalities (i.e., $\gamma \in \mathcal{D}_{\gamma}$ ) and one linear equality (i.e., $\left.\sum_{k=1}^{n} \gamma_{k} x_{t}^{k}=0\right)$ in the unknown $\gamma \in R^{n}$. It is also seen that the search can be started from the unique real root of one nominal polynomial obtained, e.g., setting $\gamma=\gamma^{c}$; next, only right side of the nominal root of equation (36) and only left side of the nominal root of equation (37) are explored in order to find a suitable approximation of $\bar{x}$ and $\underline{x}$ respectively. Analogous considerations can be made in all other cases $\left(\gamma_{n}>0, \gamma_{n}<0, n\right.$ odd, $n$ even).

4.2 Input sequence design - One is left with the problem of the choice of the input sequence $\left\{u_{t}\right\}$. In order to drive the inner signal $\left\{x_{t}\right\}$ into the desired interval, the input signal $\left\{u_{t}\right\}$ should contain a DC component $u_{D C}$ (offset) and a dynamic exciting signal $\left\{u_{t d}\right\}$ whose amplitudes should be chosen in such a way that $x_{t}=x_{D C}+x_{t d}$ satisfies either (31) $\forall t$ or (32) $\forall t$. Since the steady-state gain of the linear subsystem is constrained to be one, the amplitudes of the DC components in $u_{t}=u_{D C}+u_{t d}$ and $x_{t}$ are the same, i.e., $u_{D C}=x_{D C}$. Guidelines for the design of the dynamic exciting signal $\left\{u_{t d}\right\}$ are provided by the following two propositions.

Proposition 2. For given $u_{D C} \geq \bar{x}$, the sequence $\left\{x_{t}\right\}$ satisfies (31) if and only if:

$$
\left\|\left\{u_{t d}\right\}\right\|_{\infty} \leq \frac{\left|u_{D C}-\bar{x}\right|}{\|g\|_{1}}
$$

where $g$ and $\|g\|_{1}$ are, respectively, the impulse response and the $\ell_{1}$ norm of the linear block; $\|\cdot\|_{\infty}$ is the $\ell_{\infty}$ norm of a sequence.

Proposition 3. For given $u_{D C} \leq \underline{x}$, the sequence $\left\{x_{t}\right\}$ satisfies (32) if and only if:

$$
\left\|\left\{u_{t d}\right\}\right\|_{\infty} \leq \frac{\left|u_{D C}-\underline{x}\right|}{\|g\|_{1}}
$$

Propositions 2 and 3 are straightly derived from the definition of $\ell_{\infty}$-norm $/ \ell_{\infty}$-norm system gain which equals the $\ell_{1}$-norm of $g$.

Remark 2 - Note that Proposition 2 and Proposition 3 give necessary and sufficient conditions for $\left\{w_{t}\right\}$ to satisfy either inequality (26) or (27).

Remark 3 - Note that even if only a rough upper bound $g_{u p}$ of $\|g\|_{1}$ is known, inequality (38) is satisfied by choosing an input dynamic exciting sequence $\left\{u_{t d}\right\}$ such that

$$
\left\|\left\{u_{t d}\right\}\right\|_{\infty} \leq \frac{\left|u_{D C}-\bar{x}\right|}{g_{u p}}
$$

while, (39) is satisfied by choosing an input dynamic exciting sequence $\left\{u_{t d}\right\}$ such that

$$
\left\|\left\{u_{t d}\right\}\right\|_{\infty} \leq \frac{\left|u_{D C}-\underline{x}\right|}{g_{u p}}
$$

Otherwise, when no a priori information on the $\ell_{1}$ norm of the linear systems is available, inequalities (38) and (39) can be indirectly satisfied varying the amplitude of the sequence $\left\{u_{t d}\right\}$ by trial and error until inequalities (26) and (27) are met by the output sequence $\left\{w_{t}\right\}$, i.e., until the measured output sequence $\left\{y_{t}\right\}$ satisfies the following inequalities $\forall t$ :

$\left(y_{t}-\Delta \eta_{t}\right)>\bar{w}$ or $\left(y_{t}+\Delta \eta_{t}\right)<\underline{w}$, when $n$ is odd

or

$$
\begin{aligned}
& \operatorname{sign}\left(\gamma_{n}\right)\left(y_{t}-\operatorname{sign}\left(\gamma_{n}\right) \Delta \eta_{t}\right)>\frac{1+\operatorname{sign}\left(\gamma_{n}\right)}{2} \bar{w}+ \\
& -\frac{1-\operatorname{sign}\left(\gamma_{n}\right)}{2} \underline{w}, \text { when } n \text { is even }
\end{aligned}
$$

\section{EVALUATION OF BOUNDS ON THE UNMEASURABLE INNER SIGNAL.}

Given the polynomial nonlinearity as characterized in Section 3 and a feasible sequence of measured outputs $\left\{y_{t}\right\}$ as characterized in Section 4, 
in this section it is shown how upper and lower bounds on the unmeasurable inner signal $x_{t}$ are evaluated. Such bounds, together with the input sequence $u_{t}$ will be used to bound the parameters of the linear dynamic block in Section 6. Combining equations (5), (6) and (7) one obtains

$$
\left|y_{t}-\sum_{k=1}^{n} \gamma_{k} x_{t}^{k}\right| \leq \Delta \eta_{t}, \quad t=1,2, \ldots, N
$$

Given the output measurement $y_{t}$, its uncertainty bounds $\Delta \eta_{t}$ and the feasible parameter set $\mathcal{D}_{\gamma}$, upper and lower bounds on the unmeasurable inner signal $x_{t}$ are defined as

$$
\begin{aligned}
x_{t}^{\max } & =\max \left\{x_{t} \in R: y_{t}-\sum_{k=1}^{n} \gamma_{k} x_{t}^{k} \leq \Delta \eta_{t},\right. \\
& \left.y_{t}-\sum_{k=1}^{n} \gamma_{k} x_{t}^{k} \geq-\Delta \eta_{t}, \text { for some } \gamma \in \mathcal{D}_{\gamma}\right\}
\end{aligned}
$$

$$
\begin{aligned}
x_{t}^{\text {min }} & =\min \left\{x_{t} \in R: y_{t}-\sum_{k=1}^{n} \gamma_{k} x_{t}^{k} \leq \Delta \eta_{t},\right. \\
& \left.y_{t}-\sum_{k=1}^{n} \gamma_{k} x_{t}^{k} \geq-\Delta \eta_{t}, \text { for some } \gamma \in \mathcal{D}_{\gamma}\right\}
\end{aligned}
$$

Equations (45) and (46) show nonlinear relations in the unknown $x_{t}$ and the uncertain $\gamma$. The computation of $x_{t}^{\max }$ and $x_{t}^{\text {min }}$ can be carried out through a one dimensional searching procedure. It is noticed that for a given $x_{t} \in R$, in order to solve each one of the above equation one must solve a set of $2 M+2$ linear inequalities. It is also seen that the search can be started from the unique real root of one nominal polynomial obtained, e.g., setting $\gamma=\gamma^{c}$ and $\Delta \eta=0$; next, right side and left side of that root are explored to find $x_{t}^{\max }$ and $x_{t}^{\min }$ respectively. If the following quantities are defined

$$
\begin{gathered}
x_{t}^{c}=\frac{x_{t}^{\text {min }}+x_{t}^{\text {max }}}{2} \\
\Delta x_{t}=\frac{x_{t}^{\text {max }}-x_{t}^{\text {min }}}{2}
\end{gathered}
$$

a compact description of $x_{t}$ in terms of $x_{t}^{c}$ and $\delta x_{t}$ is as follows

$$
\begin{aligned}
& x_{t}=x_{t}^{c}+\delta x_{t} \\
& \left|\delta x_{t}\right| \leq \Delta x_{t} .
\end{aligned}
$$

\section{BOUNDING THE PARAMETERS OF THE LINEAR DYNAMIC MODEL}

In this section bounds on the parameters of the linear dynamic block are evaluated. The identification of the linear block can be formulated in the frame of output error models, i.e., in terms of the known input sequence $\left\{u_{t}\right\}$ and the uncertain inner sequence $\left\{x_{t}\right\}$ as shown in Figure 3. Combining equation (2), (3), (4) and (49) one gets

$x_{t}^{c}=-\sum_{i=1}^{n a}\left(x_{t-i}^{c}-\delta x_{t-i}\right) a_{i}+\sum_{j=0}^{n b} u_{t-j} b_{j}+\delta x_{t}$.
The feasible parameter region for the linear system is defined as

$$
\begin{gathered}
\mathcal{D}_{\theta}=\left\{\theta \in R^{p}: A\left(q^{-1}\right)\left[x_{t}^{c}-\delta x_{t}\right]=B\left(q^{-1}\right) u_{t} ;\right. \\
\left.g=1 ;\left|\delta x_{t}\right| \leq \Delta x_{t} ; t=1, \ldots, N\right\} .
\end{gathered}
$$

Due to serial dependence between $x_{t}$ samples at different time, exact parameter bounds for model (51) are no longer linear (Veres and Norton, 1991). Thus, in this paper, a polytopic outer approximation $\mathcal{D}_{\theta}^{\prime}$ of the exact $F P R \mathcal{D}_{\theta}$, i.e. $\mathcal{D}_{\theta}^{\prime} \supset$ $\mathcal{D}_{\theta}$, will be presented, together with an orthotopeouter bounding set $\mathcal{B}_{\theta}$ of $\mathcal{D}_{\theta}^{\prime}$, which provides parameters uncertainties intervals.

The dynamic model (51) with bounds on the inner signal uncertainty $\delta x_{t}$ (50) fits in the framework of the bounded output error model outlined by Clement and Gentil (1988) and in the more general framework of the bounded-errors-in-variables model (see, e.g. Cerone, 1993; Veres and Norton, 1991). Indeed, the feasible parameter region $\mathcal{D}_{\theta}^{\prime}$ can be described by

$$
\begin{aligned}
& \left(\phi_{t}-\Delta \phi_{t}\right)^{\mathrm{T}} \theta \leq y_{t}+\Delta \eta_{t} \\
& \left(\phi_{t}+\Delta \phi_{t}\right)^{\mathrm{T}} \theta \geq y_{t}-\Delta \eta_{t}
\end{aligned}
$$

where

$$
\begin{gathered}
\phi_{t}^{\mathrm{T}}=\left[\begin{array}{llll}
-x_{t-1}^{c} \ldots-x_{t-n a}^{c} & u_{t} & u_{t-1} \ldots & u_{t-n b}
\end{array}\right] \\
\Delta \phi_{t}^{\mathrm{T}}=\left[\begin{array}{lllll}
\Delta x_{t-1} \operatorname{sgn}\left(a_{1}\right) & \ldots & \Delta x_{t-n a} \operatorname{sgn}\left(a_{n a}\right) \\
& \left.\begin{array}{cccc}
0 & 0 & \ldots & 0
\end{array}\right]
\end{array}\right.
\end{gathered}
$$

A further significant reduction of $\mathcal{D}_{\theta}^{\prime}$ is obtained adding the constraint about the steady-state gain. As a matter of fact, equation (10), which can be written in the following form

$$
\left[\begin{array}{lllllll}
1 & \ldots & 1 & -1 & -1 & \ldots & -1
\end{array}\right] \theta=-1
$$

forces the feasible parameter region to belong to the hyperplane described by (57).

The orthotope-outer bounding set $\mathcal{B}_{\theta}$ is defined as

$$
\begin{gathered}
\mathcal{B}_{\theta}=\left\{\theta \in R^{p}: \theta_{j}=\theta_{j}^{c}+\delta \theta_{j},\right. \\
\left.\left|\delta \theta_{j}\right| \leq \Delta \theta_{j} / 2, j=1, \ldots, p\right\},
\end{gathered}
$$

where

$$
\begin{gathered}
\theta_{j}^{c}=\frac{\theta_{j}^{\text {min }}+\theta_{j}^{\text {max }}}{2}, \\
\Delta \theta_{j}=\left|\theta_{j}^{\text {max }}-\theta_{j}^{\text {min }}\right|,
\end{gathered}
$$

and

$$
\theta_{j}^{\min }=\min _{\theta \in \mathcal{D}_{\theta}^{\prime}} \theta_{j}, \quad \theta_{j}^{\max }=\max _{\theta \in \mathcal{D}_{\theta}^{\prime}} \theta_{j} .
$$

Parameter vectors $\gamma^{c}$ and $\theta^{c}$ are Chebishev centers in the $\ell_{\infty}$ norm of $\mathcal{D}_{\gamma}$ and $\mathcal{D}_{\theta}^{\prime}$ respectively and are commonly referred to as central estimates.

\section{CONCLUSIONS}

In this paper the identification of SISO Wiener models has been considered when the nonlinear block can be modeled by a polynomial, with finite and known order, and when the output measurements are corrupted by unknown but bounded noise. First, using steady-state input-output data, parameters of the nonlinear block have been tightly bounded. Next in order to estimate the parameter of the linear block, the evaluation of 
the inner unmeasurable signal has been considered under the hypothesis of non-invertibility of the nonlinear block. Conditions under which the inner signal can be consistently estimated by inputoutput data have been established and, on the basis of such conditions, the design of a suitable input sequence has been outlined. Then, through a dynamic experiment, upper and lower bounds on the inner signal have been computed. Finally, such bounds, together with the input sequence, have been used for bounding the parameters of the linear block.

\section{ACKNOWLEDGMENTS}

This research was partly supported by the italian Ministry of Universities and Research in Science and Technology (MURST), under the plan "Robustness techniques for control of uncertain systems".

\section{REFERENCES}

Bai, E.W. (2002). A blind approach to the Hammerstein - Wiener model identification. Automatica 38, 967-979.

Billings, S.A. (1980). Identification of nonlinear systems - a survey. IEE Proc. Part $D$ $\mathbf{1 2 7}(6), 272-285$.

Billings, S.A. and S.Y. Fakhouri (1977). Identification of nonlinear systems using Wiener model. Electron. Lett. 17, 502-504.

Cerone, V. (1993). Feasible parameter set for linear models with bounded errors in all variable. Automatica 29(6), 1551-1555.

Cerone, V. M. Milanese and D. Regruto (2002). Parameters bounds for Wiener models. Internal Report DAI0215.

Clement, T. and S. Gentil (1988). Recursive membership set estimation for armax models: an output-error approach. In: Proc. 12th IMACS Congress on Scientific Computation. pp. 484486.

Haber, R. and H. Unbehauen (1990). Structure identification of nonlinear dynamic systems a survey on input/uotput approaches. Automatica 26(4), 651-677.

Kalafatis, A.D. L. Wang and W.R. Cluett (1997). Identification of Wiener-type nonlinear systems in a noisy enviroment. Int. J. Control 66(6), 923-941.

Krzyżak, A. (1993). Identification of nonlinear block-oriented systems by the recursive kernel estimate. Int. J. Franklin Inst. 330(3), 605627.

Lang, Z.Q. (1993). Controller design oriented model identification method for Hammerstein system. Automatica 29(3), 767-771.

Milanese, M. and A. Vicino (1991). Optimal estimation theory for dynamic sistems with set membership uncertainty: an overview. Automatica 27(6), 997-1009.

Milanese, M. Norton, J. Piet-Lahanier, H. and Walter, E. Eds.) (1996). Bounding approaches to system identification. Plenum Press. New York.

Norton(Ed.), J.P. (1994). Special issue on bounded-error estimation. Int. J. of Adaptive Control \&6 Signal Processing.

Norton(Ed.), J.P. (1995). Special issue on bounded-error estimation. Int. J. of Adaptive Control 8 Signal Processing.
Stoica, P. and T. Söderström (1982). Instrumental-variable methods for identification of hammerstein systems. Int. J. Control 35(3), 459-476.

Sun, L. W. Liu and A. Sano (1999). Identification of a dynamical system with input nonlinearity. IEE Proc. Part D 146(1), 41-51.

Veres, S.M. and J.P. Norton (1991). Parameterbounding algorithms for linear errors in variables models. In: Proc. of IFAC/IFORS Symposium on Identification and System Parameter Estimation. pp. 1038-1043.

Walter, E. and H. Piet-Lahanier (1990). Estimation of parameter bounds from bounded-error data: a survey. Mathematics and Computers in simulation 32, 449-468.

Wigren, T. (1998). Output error convergence of adaptive filters with compensation for output nonlinearities. IEEE Trans. Automatic. Control 43(7), 975-978.

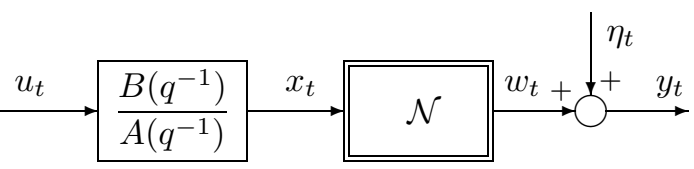

Fig. 1. Single-input single-output Wiener model.

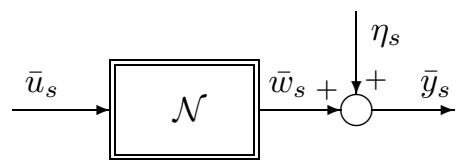

Fig. 2. Steady-state behaviour of the Wiener model when $g=1$.

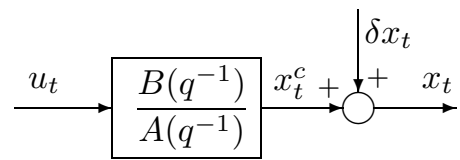

Fig. 3. Output error set-up for bounding the parameters of the linear system. 